Editorial

\title{
Patient Positioning during Neurosurgery: A Relevant Skill for Neuroanesthesiologist in a Multidisciplinary Team Work
}

\author{
Federico Bilotta ${ }^{1}$ Paola G. Sergi ${ }^{1}$ Valeria Spennati ${ }^{1}$ \\ ${ }^{1}$ Department of Anesthesiology, Critical Care and Pain Medicine, \\ 'Sapienza' University of Rome, Rome, Italy \\ J Neuroanaesthesiol Crit Care 2020;7:49-51
}

Neuroanesthesia encompasses clinical management of patients undergoing brain and spine procedures that might require positioning which are not usual in other specialties, and these include the following: prone, lateral (and park bench) and semilateral, as well as sitting and semisitting. ${ }^{1}$ Complications related with patient positioning in neurosurgery undergoing cerebral and spinal procedures is a major concern extensively addressed by Rozet and Vavilala in a seminal review. ${ }^{2}$ Rozet and Vavilala report tips on head and body positioning during brain surgery, and related risks in the perioperative period and how to prevent them. Risks related to prone positioning include the following: pressure sores, vascular compression, brachial plexus injuries, air embolism, blindness, and spinal cord damage due to vascular or nerve damage, with related peripheral limbs deficit and even quadriplegia. Insights and complications related to the use of sitting position include venous air embolism, paradoxical air embolism, bradycardia or cardiac arrest. The review articles from Boyle et al and Goraksha et al, included in this issue, address appropriate positioning of neurosurgical patients and also provide new and relevant information in this field. ${ }^{3,4}$

Boyle and colleagues completed a systematic review that reported evidence relevant for intraoperative positioning in patients undergoing cervical spine surgery with posterior approach. ${ }^{3}$ In their review, a total of 9875 studies were retrieved and 20 were selected as appropriate for data analysis. The literature review identified three methods of prone positioning of patients with cervical pathology: logroll with manual in-line stabilization (MILS), "sandwich and flip" technique rotation of the patient using a specialized spinal table, or awake prone positioning. During MILS positioning, one person holds patient's head in line with the shoulders, while at least three other team members roll the patient from the supine to the prone position. During the "sandwich and flip" technique, the operators can use different types of spinal surgery tables (Jackson, Allen, and Galen spinal table). These tables have a built-in rotation mechanism that allows the patient to be positioned supine and induced on the flat board. The carbon frame is then placed on top of the patient (sandwiching them in position), and the arms and legs are secured with safety straps. In patients undergoing spine procedures, awake self-positioning of a patient is another alternative that allows rapid neurological assessment after repositioning. In these patients, two techniques of awake prone positioning have been reported in the literature: the first method involves securing the airway while the patient is awake, and then the patient moves himself or herself into the prone position; the second technique involves positioning the patient prone first and then the airway is secured using either a fiber-optic intubation or a laryngeal mask airway. ${ }^{5}$ The major advantage of MILS approach is that it requires no additional equipment, making the maneuver faster and more efficient to perform, but the main limitation is the difficulty of achieving precise and coordinated movement by all members of the team to maintain the spine inline during positioning. The "sandwich and flip" rotation was associated with over $50 \%$ reduction in both flexion-extension and axial-lateral rotation as compared with logroll with MILS, so this approach resulted in the safest way to position a patient with cervical pathology into a prone stance for surgery. Awake self-positioning resulted to be a good alternative in patients who are physically capable but securing the airway once the patient is in the prone position is technically more difficult.

Goraksha and colleagues, in their review article, describe the potential complications associated with the sitting position in neurosurgical patients. ${ }^{4}$ The authors highlight how the collected evidence prove that to ensure patient safety and a successful outcome, a thorough preoperative anesthetic evaluation to decide if the sitting position is suitable for the patient is essential. As in any neurosurgical case, all

\footnotetext{
Address for correspondence

Federico Bilotta, MD, PhD, Department of Anesthesiology, Critical Care and Pain Medicine, 'Sapienza' University of Rome, Via Acherusio 16, Rome 00199, Italy (e-mail: bilotta@tiscali.it).
}

DoI https://doi.org/ 10.1055/s-0040-1712082 ISSN 2348-0548.
(C)2020 Indian Society of

Neuroanaesthesiology and Critical Care
License terms

(ㄷ) (1) $\ominus \circledast$ 
patients need to be evaluated preoperatively for physical and neurological status, as well as blood and other pertinent investigations.

One of the advantages of the sitting position is decreased blood loss due to drainage of cerebral venous blood away from the operating site. A furthermore complicated issue related to the use of sitting position is the tension pneumocephalus. ${ }^{5}$ The incidence of postoperative pneumocephalus in sitting position may reach $100 \%$, which may be due to negative cerebral spinal fluid pressure and/or residual air during closure of the dura. The review from Goraksha and colleagues contributes to the long-lasting and controversial confrontation on sitting positioning (in terms of complication: postural hypotension, venous air embolism, etc.), that with thorough preoperative anesthetic evaluation, use of advanced intraoperative monitoring, and meticulous surgical techniques, can be safely used in indicated cases.

Several potential threats can arise from inappropriate positioning, and the ability to appropriately convey the patient of intraoperative stance, which better fits the surgical requirements, is among the specific skill that qualify competence in neuroanesthesia. ${ }^{6,7}$ The process can be time consuming and for this reason some centers have minimized the possible choices that surgeons can pick up. ${ }^{8}$ On the other hand, several centers have banned some intraoperative positioning, as the sitting and semisitting, for the inherent risks., ${ }^{2,89}$ In neurosurgical-neuroanesthesia teams that have developed a high-level of cooperation, this phase of the procedure is characterized by an attentive and interactive participation of two professional surgical nurses (who are often dedicated). ${ }^{7}$ Potential risks associated with incorrect positioning relate to vascular and nerve injuries that can be prevented with adequate inspection of the major plexuses; postoperative blindness is reported to occur after prone positioning (intraoperative anemia, arterial hypotension and eye compression are among the modifiable risk factors); venous stasis, with suboptimal surgical field exposure, can complicate both cerebral (should jugular and neck venous drainage be suboptimal) and spinal prone cases (when chest or abdominal compression exert and increase in the venous pressure). ${ }^{10-13}$ Blood flow in vertebral and brain arteries (resulting in quadriparesis and quadriplegia) may significantly decrease in patients with thoracic compression and increased intrathoracic pressure; also, the hyperflexion of the head and neck can contribute to venous stasis. ${ }^{7}$ Furthermore, the use of PEEP (positive end-expiratory pressure) during neurosurgical procedures performed in the sitting position carries controversial effects due to reduced venous return and cardiac output. Physiological PEEP at levels not higher than 4 to $5 \mathrm{~cm} \mathrm{H}_{2} \mathrm{O}$ contributes to maintaining the increase in the transthoracic pressure-that is transmitted to the venous system and turns into an increase in central venous pressure (CVP)-within acceptable values, thus ensuring effective venous drainage. ${ }^{7}$ Interestingly, major neurological deficit can complicate also the course of non-neurosurgical patients undergoing general surgery due to pre-existing and unknown cervical spondylosis, and are referable to incorrect positioning or neck stretching during laryngoscopy. ${ }^{14}$ More recently, a systematic review on prevention of intraoperative bleeding during procedures in prone position highlighted that specific support frames (such as jackknife position) minimize hemodynamic changes and intraoperative blood loss. ${ }^{15}$

In conclusion, the papers by Boyle et al and Goraksha et al bring the attention of neuroanesthesia community to a well-established aspect of our clinical practice: optimal intraoperative patient positioning for neurosurgical procedures. The related competence should not only prevent-to the largest extent possible-positioning lesions (stretching of neurovascular plexuses and postoperative loss of vision) but also improve the quality of intraoperative surgical field exposure (optimal venous drainage and minimal thoracic compression) and prevent surgical-related intraoperative complications (venous air embolism). This complex and relevant task should be achieved through interactive teamwork that includes the proactive role of the neuroanesthesiologists.

\section{Conflict of interest}

None declared.

\section{References}

1 Clatterbuck R, Tamargo R. Surgical positioning and exposures for cranial procedures. In: Winn H, ed. Youmans Neurological Surgery. 5th ed. Philadelphia, Pennsylvania: Sounders Elsevier Inc; 2004;623-645

2 Rozet I, Vavilala MS. Risks and benefits of patient positioning during neurosurgical care. Anesthesiol Clin 2007;25(3): 631-653

3 Boyle SL, Unger Z, Kulkarni V, Massicotte EM, Venkatraghavan L. Prone positioning of patients with cervical spine pathology. J Neuroanaesth Crit Care 2020;7(2):70-76

4 Goraksha S, Thakore B, Monteiro J. Sitting position in neurosurgery. J Neuroanaesth Crit Care 2020;7(2):77-83

5 Olsen KS, Petersen JT, Pedersen NA, Rovsing L. Self-positioning followed by induction of anaesthesia and insertion of a laryngeal mask airway versus endotracheal intubation and subsequent positioning for spinal surgery in the prone position: a randomised clinical trial. Eur J Anaesthesiol 2014;31(5): 259-265

6 Gruenbaum SE, Bilotta F. Neuroanesthesiology: building the path to superior clinical care through research and education. Curr Opin Anaesthesiol 2018;31(5):499-500

7 Bilotta F. Neuroanesthesiology: the ineludible path toward super-specialty. Curr Opin Anaesthesiol 2017;30(5):525-526

8 Builes-Aguilar A, Diaz-Gomez JL, Bilotta F. Education in neuroanesthesia and neurocritical care: trends, challenges and advancements. Curr Opin Anaesthesiol 2018;31(5):520-525

9 Srivastava S. The sitting position for neurosurgery: a bane or a boon. Neurol India 2018;66(1):223-225

10 Gracia I, Fabregas N. Craniotomy in sitting position: anesthesiology management. Curr Opin Anaesthesiol 2014;27(5): 474-483

11 Epstein NE. Perioperative visual loss following prone spinal surgery: a review. Surg Neurol Int 2016;7(suppl 13): S347-S360

12 Edgcombe H, Carter K, Yarrow S. Anaesthesia in the prone position. Br J Anaesth 2008;100(2):165-183 
13 Rath GP, Bithal PK, Chaturvedi A, Dash HH. Complications related to positioning in posterior fossa craniectomy. J Clin Neurosci 2007;14(6):520-525

14 Mathkour M, McCormack E, Hanna J, et al. Iatrogenic spinal cord injury with tetraplegia after an elective non-spine surgery with underlying undiagnosed cervical spondylotic myelopathy: Literature review and case report. Clin Neurol Neurosurg 2019;187:105549

15 Willner D, Spennati V, Stohl S, Tosti G, Aloisio S, Bilotta F. Spine surgery and blood loss: systematic review of clinical evidence. Anesth Analg 2016;123(5):1307-1315 